\title{
A note on the representation and definition of semiseparable matrices
}

\author{
Raf Vandebril \\ Marc Van Barel \\ Nicola Mastronardi
}

Report TW 368, October 2003

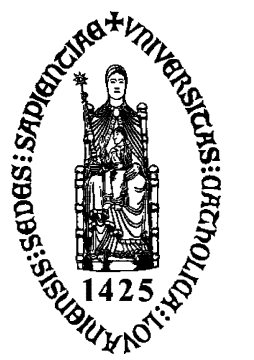

\section{Katholieke Universiteit Leuven}

Department of Computer Science

Celestijnenlaan 200A - B-3001 Heverlee (Belgium) 


\title{
A note on the representation and definition of semiseparable matrices
}

\author{
Raf Vandebril \\ Marc Van Barel \\ Nicola Mastronardi \\ Report TW 368, October 2003 \\ Department of Computer Science, K.U.Leuven
}

\begin{abstract}
In this paper the definition of semiseparable matrices is investigated. Properties of the frequently used definition and the corresponding representation by generators are deduced. Corresponding to the class of tridiagonal matrices another definition of semiseparable matrices is introduced preserving the nice properties dual to the class of tridiagonal matrices. Several theorems and properties are included showing the viability of this alternative definition.

Because of the alternative definition, the standard representation of semiseparable matrices is not satisfying anymore. The concept of a representation is explicitely formulated and a new kind of representation corresponding to the alternative definition is given. It is proved that this representation keeps all the interesting properties of the generator representation.

As an example of the effectivity of the new representation, we design on $O(n)$ algorithm for the multiplication of a semiseparable matrix given by the new representation, with a vector.
\end{abstract}

Keywords : semiseparable matrices, representations, definitions AMS(MOS) Classification : Primary : 15A21, Secondary : 65F30. 


\title{
A note on the representation and definition of semiseparable matrices *
}

\author{
Raf Vandebril † Marc Van Barel $\ddagger$ Nicola Mastronardi §
}

6th November 2003

\begin{abstract}
In this paper the definition of semiseparable matrices is investigated. Properties of the frequently used definition and the corresponding representation by generators are deduced. Corresponding to the class of tridiagonal matrices another definition of semiseparable matrices is introduced preserving the nice properties dual to the class of tridiagonal matrices. Several theorems and properties are included showing the viability of this alternative definition.

Because of the alternative definition, the standard representation of semiseparable matrices is not satisfying anymore. The concept of a representation is explicitely formulated and a new kind of representation corresponding to the alternative definition is given. It is proved that this representation keeps all the interesting properties of the generator representation.

As an example of the effectivity of the new representation, we design on $O(n)$ algorithm for the multiplication of a semiseparable matrix given by the new representation, with a vector.
\end{abstract}

Keywords: semiseparable matrices, representations, definitions

\section{Introduction}

To our knowledge the most early papers concerning semiseparable matrices are the papers $[1,2,13,21,35,36,37,38]$. In these papers semiseparable matrices are defined

\footnotetext{
${ }^{*}$ The research of the first two authors was supported by the Research Council K.U.Leuven, project OT/00/16 (SLAP: Structured Linear Algebra Package), by the Fund for Scientific Research-Flanders (Belgium), projects G.0078.01 (SMA: Structured Matrices and their Applications), G.0176.02 (ANCILA: Asymptotic aNalysis of the Convergence behavior of Iterative methods in numerical Linear Algebra), and G.0184.02 (CORFU: Constructive study of Orthogonal Rational Functions), by the K.U.Leuven (Bijzonder Onderzoeksfonds), and by the Belgian Programme on Interuniversity Poles of Attraction, initiated by the Belgian State, Prime Minister's Office for Science, Technology and Culture, project IUAP V-22 (Dynamical Systems and Control: Computation, Identification \& Modelling). The research of the third author was partially supported by MIUR, grant number 2002014121 (third author). The scientific responsibility rests with the authors

†Email: raf.vandebril@cs.kuleuven.ac.be

†Email: marc.vanbarel@cs.kuleuven.ac.be

${ }^{\S}$ Email: irmanm21@area.ba.cnr.it
} 
as the inverses of unreducible tridiagonal matrices. In these latter papers the authors refer to these matrices as: Green's matrix, one-pair matrix, single-pair matrix. In these papers different proofs are included stating the fact that the inverses of unreducible band matrices are semiseparable matrices, also singular and higher order semiseparable matrices are considered.

Semiseparable matrices appear in several types of applications, e.g., the field of integral equations [24, 27, 28], boundary value problems [26, 24, 30, 39], in the theory of Gauss-Markov processes [29], time varying linear systems [10, 23], in statistics [25], acoustic and electromagnetic scattering theory [9] and rational interpolation [40].

Also lots of connections appear within the field of linear algebra, already lots of algorithms for different types of factorizations, reductions, solvers,... are developed. For example there exist reduction algorithms to reduce semiseparable matrices towards bidiagonal or tridiagonal matrices [17, 32], also reduction algorithms based on the divide and conquer approach exist [31]. Recently there is also a reduction algorithm in the other direction namely to transform symmetric matrices into semiseparable ones [41]. Also several papers are dedicated to different types of factorizations algorithms and the solvers corresponding to these factorizations [6, 12, 42]. Extension of this theory leads to solvers for higher rank semiseparable matrices plus banded matrices [8, 22]. There exist also techniques for solving least squares problems for semiseparable matrices [3], and also rank revealing problems via semiseparable matrices [33].

There are several theoretical papers investigating properties of this class of matrices $[14,18,20,19]$. Inversion formulas for diagonal plus semiseparable matrices are studied in [11], properties of possibly singular semiseparable matrices are investigated in [16]. Recently a new class of semiseparable matrices was introduced called recursively or sequentially semiseparable matrices. For example the papers $[4,6]$ introduce algorithms for this new, useful class of matrices.

Also algorithms have been designed to compute the eigenvalues and singular values of semiseparable matrices or semiseparable plus banded matrices, via divide and conquer algorithms and via the reduction towards bi or tridiagonal form [7, 15, 5, 34]. Recently also algorithms have been proposed for $Q R$ algorithms working directly on the semiseparable matrix [42, 43].

This paper consists of three main parts. In a first part the often used definition of semiseparable matrices, namely by generators, is investigated. Because of the strong connection with tridiagonal matrices, we based ourself on the class of tridiagonal matrices to devise properties for the semiseparable matrices. The result of this part is the definition of a larger class of semiseparable matrices, containing the former class. The second part of this paper investigates a new representation for this wider class. We search for a representation keeping all the interesting properties of the generator representation. In a final smaller part, an algorithm is given showing that it is possible to multiply a semiseparable matrix, in Givens-vector representation, with a vector in order $O(n)$ operations. 


\section{Semiseparable matrices and their definition}

In this section the definition of a semiseparable matrix is investigated. We start with one of the first definitions, namely as the inverse of unreducible tridiagonal matrices. Properties of this class of semiseparable matrices are compared with the properties of the class of tridiagonal matrices. Based on these comparisons an alternative definition of semiseparable matrices is given. Note that this paper is restricted to the class of symmetric matrices. The extension to higher order and nonsymmetric semiseparable matrices is straightforward.

\subsection{One-pair matrices as the inverse of tridiagonal matrices}

Originally, semiseparable matrices were defined as the inverses of unreducible tridiagonal matrices, see for instance $[1,36]$. Later on they were also defined as the inverses of unreducible band matrices [21]. Note that these early definitions immediately exclude the possibility of having singular semiseparable matrices. In the book [21] semiseparable matrices got for the first time a name, namely one-pair or single-pair matrices (depending on the translation; the book was originally written in Russian). In fact these one-pair matrices are a special sort of semiseparable matrices, and they have strong connections with Jacobi matrices, more commonly known as tridiagonal matrices. In this section the two most important theorems of the book [21] will be mentioned, and their proofs will be given in appendix A.

Originally the one-pair matrices were defined as:

Definition 1 A one-pair matrix is a symmetric matrix $S$ such that

$$
S_{i, j}= \begin{cases}u_{i} v_{j} & (i \leq j) \\ u_{j} v_{i} & (i \geq j)\end{cases}
$$

where the elements $u_{i}$ and $v_{j}$ are chosen arbitrarily.

The main theorem in [21, Chapter II] states that the inverse of an unreducible Jacobi matrix is a one-pair matrix.

Theorem 1.1 Suppose A is a symmetric tridiagonal matrix of size n:

$$
\left(\begin{array}{ccccc}
a_{1} & b_{1} & & & \\
b_{1} & a_{2} & b_{2} & & \\
& b_{2} & a_{3} & \ddots & \\
& & \ddots & \ddots & b_{n-1} \\
& & & b_{n-1} & a_{n}
\end{array}\right)
$$

with all the $b_{i}$ different from zero. The inverse of $A$ will be a one-pair matrix.

PROOF: See appendix A.

This theorem states that the inverse of an unreducible symmetric tridiagonal matrix is a so called one-pair matrix, nothing is mentioned about general tridiagonal matrices.

Also the inverse of the preceding theorem is true: the inverse of an invertible onepair matrix is a tridiagonal matrix, with nonzero subdiagonal elements. The proof is given in appendix A. 


\subsection{Symmetric tridiagonal matrices}

Before introducing another definition of a semiseparable matrix, we take a close look at the class of symmetric tridiagonal matrices and derive some of its properties. We consider this class of matrices, because the inverse of an invertible symmetric tridiagonal is a semiseparable matrix. Therefore, both classes of matrices have very strong connections.

A first important property of symmetric trididiagonal matrices, is that they are defined by the diagonal and subdiagonal elements, in fact storing $2 n-1$ elements is enough to reconstruct the tridiagonal matrix. A second important point is the fact that also singular matrices can be tridiagonal and symmetric. The following property is important in several applications. Suppose we have a symmetric tridiagonal matrix $T$ and apply the $Q R$ algorithm, to compute the eigenvalues, to this matrix. Doing so we get a sequence of tridiagonal matrices

$$
T^{(0)} \rightarrow T^{(1)} \rightarrow \cdots \rightarrow T^{(n)} \rightarrow \cdots
$$

which converge towards a (block-)diagonal matrix. This (block-)diagonal matrix also belongs to the class of tridiagonal matrices. To formulate this more precisely, we need the definition of pointwise convergence:

Definition 2 The pointwise limit of a collection of matrices $A_{\varepsilon} \in \mathbb{R}^{n \times n}$ (if it exists) for $\varepsilon \rightarrow \varepsilon_{0}$, with $\varepsilon, \varepsilon_{0} \in \mathbb{R}$ and with the matrices $A_{\varepsilon}$ as

$$
A_{\varepsilon}=\left(\begin{array}{ccc}
\left(a_{1,1}\right)_{\varepsilon} & \cdots & \left(a_{1, n}\right)_{\varepsilon} \\
\vdots & \ddots & \vdots \\
\left(a_{n, 1}\right)_{\varepsilon} & \cdots & \left(a_{n, n}\right)_{\varepsilon}
\end{array}\right)
$$

is defined as:

$$
\lim _{\varepsilon \rightarrow \varepsilon_{0}} A_{\varepsilon}=\left(\begin{array}{ccc}
\lim _{\mathcal{\varepsilon} \rightarrow \varepsilon_{0}}\left(a_{1,1}\right)_{\varepsilon} & \cdots & \lim _{\mathcal{\varepsilon} \rightarrow \varepsilon_{0}}\left(a_{1, n}\right)_{\varepsilon} \\
\vdots & \ddots & \vdots \\
\lim _{\mathcal{\varepsilon} \rightarrow \varepsilon_{0}}\left(a_{n, 1}\right)_{\varepsilon} & \cdots & \lim _{\mathcal{\varepsilon} \rightarrow \varepsilon_{0}}\left(a_{n, n}\right)_{\varepsilon}
\end{array}\right)
$$

Corollary 2.1 For the class of symmetric tridiagonal matrices $T$ we have the following properties:

- They can be represented by order $O(n)$ information.

- Tridiagonal matrices can also be singular.

- The class of tridiagonal matrices is closed for pointwise convergence.

\subsection{The frequently used definition of semiseparable matrices in- vestigated}

Most of the definitions of semiseparable matrices are made by using generators, for example the papers [5, 16, 32, 42]. After given the definition we will investigate the properties of this class of semiseparable matrices in correspondence with the properties of Corollary 2.1 
Definition $3 S$ is called a semiseparable matrix of semiseparability rank $r$ if there exist two matrices $R_{1}$ and $R_{2}$ both of rank $r$, such that

$$
S=\operatorname{triu}\left(R_{1}\right)+\operatorname{tril}\left(R_{2}\right)
$$

$\operatorname{triu}\left(R_{1}\right)$ and tril $\left(R_{2}\right)$ denote respectively the strictly upper triangular part of the matrix $R_{1}$ and the lower triangular part of the matrix $R_{2}$. Suppose $r=1$, because $R_{1}$ and $R_{2}$ are now two rank one matrices, they can both be written as the outer product of two vectors, respectively $u$ and $v$ for $R_{1}$ and $s$ and $t$ for $R_{2}$. These vectors are also called the generators of the semiseparable matrix $S$.

This definition is already a generalization of the definition for one-pair matrices. First of all the matrices do not have to be symmetric anymore. Moreover semiseparable matrices of semiseparability rank higher than 1 are defined now. This definition is already much stronger than just defining semiseparable matrices as the inverses of symmetric tridiagonals. We can reconstruct the matrices by keeping $O(n)$ information. For example a symmetric semiseparable matrix of rank 1 looks like:

$$
\left(\begin{array}{ccccc}
u_{1} \cdot v_{1} & u_{1} \cdot v_{2} & u_{1} \cdot v_{3} & \ldots & u_{1} \cdot v_{n} \\
u_{1} \cdot v_{2} & u_{2} \cdot v_{2} & u_{2} \cdot v_{3} & \ldots & u_{2} \cdot v_{n} \\
u_{1} \cdot v_{3} & u_{2} \cdot v_{3} & \ddots & \ddots & \vdots \\
\vdots & \vdots & \ddots & & \\
u_{1} \cdot v_{n} & u_{2} \cdot v_{n} & \ldots & & u_{n} \cdot v_{n}
\end{array}\right)
$$

In the following part when we speak about semiseparable matrices, we mean symmetric semiseparable matrices of semiseparability rank 1 . We will also denote semiseparable matrices satisfying Definition 3 with $S(u, v)$, this means a semiseparable matrix representable with two generators $u$ and $v$. A semiseparable plus diagonal matrix is defined as the sum of a semiseparable matrix and a diagonal one.

The following example is included, to illustrate some common misunderstandings about semiseparable matrices. In several papers there are statements which are not completely true.

Example 1 Several papers state that the inverse of a tridiagonal matrix is a semiseparable matrix according to Definition 3. However, consider the following matrix:

$$
A=\left(\begin{array}{lll}
0 & 1 & 0 \\
1 & 0 & 0 \\
0 & 0 & 1
\end{array}\right)
$$

This is clearly a nonsingular, symmetric tridiagonal matrix. According to the statements above, its inverse should be a semiseparable matrix representable with two generators $u$ and $v$. Matrix $A$ is its own inverse, and one cannot represent this matrix with two generators $u$ and $v$. When we expand this class to the class of semiseparable plus diagonal matrices, we can represent the matrix $A$ in this way, but this is not the case for all the inverses of tridiagonal matrices. 
For example consider the following matrix A, which is a block combination of the matrix from above:

$$
A=\left(\begin{array}{llllll}
0 & 1 & 0 & 0 & 0 & 0 \\
1 & 0 & 0 & 0 & 0 & 0 \\
0 & 0 & 1 & 0 & 0 & 0 \\
0 & 0 & 0 & 0 & 1 & 0 \\
0 & 0 & 0 & 1 & 0 & 0 \\
0 & 0 & 0 & 0 & 0 & 1
\end{array}\right)
$$

The reader can verify that the inverse of this nonsingular symmetric tridiagonal matrix cannot be represented by two generators $u$ and $v$ nor by two generators $u, v$ and $a$ diagonal.

The following example deals with the numerical instability of the representation with generators. The reason is the non-closedness of the class of semiseparable matrices as defined in Definition 3 for the pointwise limit.

Example 2 Suppose a symmetric $5 \times 5$ matrix $A$ is given with the following eigenvalues: $\left(1,2,3,100,10^{5}\right)$. Constructing a semiseparable matrix from it (the procedure, how to do so is explained in paper [41]) generates the following matrix (Using 16 decimal digits of precision in Matlab ${ }^{1}$.):

$$
S=\left(\begin{array}{rrrrr}
1.2738 e+00 & -5.7004 e-01 & 1.2664 e-01 & -1.6459 e-04 & -7.7607 e-12 \\
-5.7004 e-01 & 2.2236 e+00 & -4.9398 e-01 & 6.4202 e-04 & 2.5203 e-12 \\
1.2664 e-01 & -4.9398 e-01 & 2.5026 e+00 & -3.2527 e-03 & -2.8150 e-12 \\
-1.6459 e-04 & 6.4202 e-04 & -3.2527 e-03 & 1.0000 e+02 & 4.8028 e-08 \\
1.5753 e-12 & -1.5858 e-13 & 1.5679 e-12 & 4.8030 e-08 & 1.0000 e+05
\end{array}\right)
$$

Even though this matrix is semiseparable it can clearly be seen that the last entry of the diagonal already approximates the largest eigenvalue. Representing this matrix with the traditional generators $u$ and $v$ gives us the following vectors:

$$
\begin{aligned}
& u=\left(\begin{array}{lllll}
8.0861 e+11 & -3.6187 e+11 & 8.0391 e+10 & -1.0448 e+08 & 1.0000 e+00
\end{array}\right)^{T} \\
& \text { and } \\
& \qquad v=\left(\begin{array}{lllll}
1.5753 e-12 & -1.5858 e-13 & 1.5679 e-12 & 4.8030 e-08 & 1.0000 e+05
\end{array}\right)^{T}
\end{aligned}
$$

Trying to reconstruct the matrix $S$ with the given generators $u$ and $v$ gives huge errors in the matrix (up to $10^{-4}$ ). This means that this representation looses almost 12 decimal digits. The digit loss in this example is unacceptable. This problem can however be explained rather easily, and it is inherent to the representation connected with the definition. Because the diagonal matrices do not belong to the class of matrices representable by the generators $u$ and $v$, the representation of the matrix above can never be very well. Very large and very small numbers can be seen in the vectors $u$ and $v$ to try to compensate the fact that the matrix is almost block diagonal.

Both examples immediately reveal problems of the representation and the definition of semiseparable matrices. First of all, not all inverses of tridiagonal matrices can be represented, and also diagonal matrices cannot be found in the class of matrices of Definition 3. Therefore an extension of this definition is needed.

\footnotetext{
${ }^{1}$ Matlab is a registered trademark of the Mathworks Inc.
} 


\subsection{An alternative new definition for semiseparable matrices}

A more general definition of a semiseparable matrix is the following one (it can easily be extended to the more general class of higher rank and nonsymmetric semiseparable matrices).

Definition 4 An $n \times n$ symmetric matrix $S$ is called a symmetric semiseparable matrix of semiseparability rank one if the following properties are satisfied:

$$
\forall i \text { with } 1 \leq i \leq n: \operatorname{rank}(S(i: n, 1: i)) \leq 1
$$

One can easily verify, that this class of matrices contains the matrices from Definition 3, diagonal matrices and also the matrices from Example 1.

In the following section some theoretical results with proofs, will justify this new definition.

\subsection{Some justifications for the alternative definition}

Assume that a semiseparable matrix satisfying Definition 4 is denoted as $S$, and a semiseparable matrix, representable with two generators $u, v$ is denoted as $S(u, v)$. The next theorem shows how the class of semiseparable matrices represented with two generators can be embedded in the class of semiseparable matrices as defined in Definition 4. To prove the theorem an interesting property is needed, revealing the close connection between the two definitions.

Proposition 4.1 Suppose a symmetric semiseparable matrix $S$ is given, which cannot be represented by two generators, then this matrix can be written as a block diagonal matrix, for which all the blocks are semiseparable matrices representable with two generators.

PROOF: It can be seen that a matrix $S$ cannot be represented by two generators (e.g. $u$ and $v$ ), if and only if

$$
\begin{array}{rll}
\exists k: 1 \leq k \leq n, \exists l: 1 \leq l \leq k & \text { such that } & S(k, l)=0 \\
\exists i: l \leq i \leq n & \text { such that } & S(i, l) \neq 0 \\
\exists j: 1 \leq j \leq k & \text { such that } & S(k, j) \neq 0 .
\end{array}
$$


(In case it is representable with two generators, a $u_{k}, v_{l}$ would exist for which one of the two has to be zero, this leads to a contradiction.)

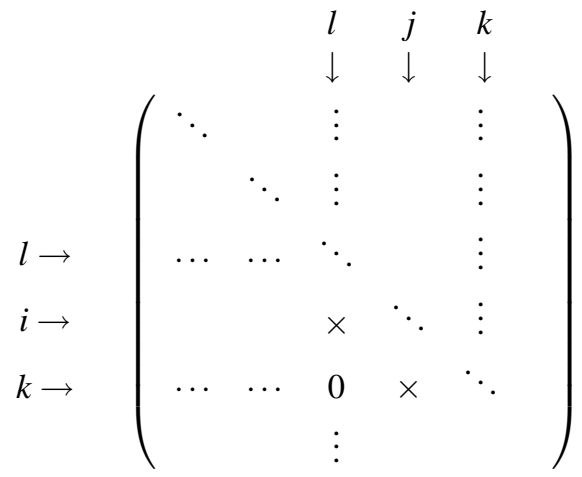

Because of the rank 1 assumption following from Definition 4, extra conditions can be placed on the indices, namely: $i<k$ and $j>l$. Suppose now, that the element $S(\hat{i}, l) \neq 0$, with $l \leq \hat{i}<k$ and all $S(i, l)=0$ for $\hat{i}<i<k$. The rank one assumption on the blocks implies that $S(i, j)=0$, for all $\hat{i}<i \leq n$ and $1 \leq j<\hat{i}+1$. This means that our matrix can be divided into two diagonal blocks. This procedure can be repeated until all the diagonal blocks are representable by two generators.

The following theorem justifies the new definition of semiseparable matrices. Also clearly seen in the following proof, is the situation when problems arise with the definition in terms of the generators.

Theorem 4.2 The pointwise closure of the class of semiseparable matrices representable by two generators is the class of semiseparable matrices according to Definition 4.

PROOF:

$\Rightarrow$ Suppose a sequence of semiseparable matrices representable with two generators is given:

$$
S(u(\varepsilon), v(\varepsilon)) \in \mathbb{R}^{n} \text { for } \varepsilon \in \mathbb{R} \text { and } \varepsilon \rightarrow \varepsilon_{0},
$$

such that the pointwise limit exists:

$$
\lim _{\varepsilon \rightarrow \varepsilon_{0}} S(u(\varepsilon), v(\varepsilon))=S \in \mathbb{R}^{n} .
$$

It will be shown that this matrix belongs to the class of semiseparable matrices from Definition 4.

It is known that $\lim _{\mathcal{\varepsilon} \rightarrow \varepsilon_{0}}\left(u_{i}(\varepsilon) v_{j}(\varepsilon)\right) \in \mathbb{R}$. (Note that this last demand does not imply that $\lim _{\varepsilon \rightarrow \varepsilon_{0}} u_{i}(\varepsilon), \lim _{\varepsilon \rightarrow \varepsilon_{0}} v_{j}(\varepsilon) \in \mathbb{R}$, which can lead to numerical unsound problems when representing these semiseparable matrices with two generators $u, v$.) It remains to prove that, $\forall i \in\{2, \ldots, n\}$ :

$$
\operatorname{rank}\left(\lim _{\varepsilon \rightarrow \varepsilon_{0}}(S(u(\varepsilon), v(\varepsilon))(i: n, 1: i))\right) \leq 1 .
$$


We have $(\forall i \in\{2, \ldots, n\})$ :

$$
\begin{aligned}
& \operatorname{rank}\left(\lim _{\varepsilon \rightarrow \varepsilon_{0}}(S(u(\varepsilon), v(\varepsilon))(i: n, 1: i))\right) \\
= & \operatorname{rank}\left(\lim _{\varepsilon \rightarrow \varepsilon_{0}}\left(u(\varepsilon)(i: n) v(\varepsilon)(1: i)^{T}\right)\right) \\
\leq & \lim _{\varepsilon \rightarrow \varepsilon_{0}}\left(\operatorname{rank}\left(u(\varepsilon)(i: n) v(\varepsilon)(1: i)^{T}\right)\right) \\
= & \lim _{\varepsilon \rightarrow \varepsilon_{0}}(\operatorname{rank}(S(u(\varepsilon), v(\varepsilon))(i: n, 1: i))) \\
= & \lim _{\varepsilon \rightarrow \varepsilon_{0}} 1=1 .
\end{aligned}
$$

Only the equality (4) needs more explanation. We will prove this by contradiction. Suppose equation (4) is not satisfied. This means that:

$$
\operatorname{rank}\left(\lim _{\varepsilon \rightarrow \varepsilon_{0}}\left(\begin{array}{cccc}
u_{i}(\varepsilon) v_{1}(\varepsilon) & u_{i}(\varepsilon) v_{2}(\varepsilon) & \ldots & u_{i}(\varepsilon) v_{i}(\varepsilon) \\
u_{i+1}(\varepsilon) v_{1}(\varepsilon) & u_{i+1}(\varepsilon) v_{2}(\varepsilon) & \ldots & u_{i+1}(\varepsilon) v_{i}(\varepsilon) \\
\vdots & \vdots & & \vdots \\
u_{n}(\varepsilon) v_{1}(\varepsilon) & u_{n}(\varepsilon) v_{2}(\varepsilon) & \ldots & u_{n}(\varepsilon) v_{i}(\varepsilon)
\end{array}\right)\right) \geq 2
$$

This means that there exist an $l$ and $k$ such that rows $l$ and $k$ are linearly independent. Suppose the following rows to be independent,

$$
\begin{gathered}
\lim _{\varepsilon \rightarrow \varepsilon_{0}}\left(u_{l}(\varepsilon) v_{1}(\varepsilon), \ldots, u_{l}(\varepsilon) v_{i}(\varepsilon)\right) \\
\lim _{\varepsilon \rightarrow \varepsilon_{0}}\left(u_{k}(\varepsilon) v_{1}(\varepsilon), \ldots, u_{k}(\varepsilon) v_{i}(\varepsilon)\right),
\end{gathered}
$$

this will lead to a contradiction.

When the latter two vectors are independent, there exist an $r, s \in\{1, \ldots, i\}$ such that the determinant of the following $2 \times 2$ matrix is different from zero:

$$
D=\operatorname{det}\left(\begin{array}{cc}
\lim _{\varepsilon \rightarrow \varepsilon_{0}} u_{l}(\varepsilon) v_{r}(\varepsilon) & \lim _{\varepsilon \rightarrow \varepsilon_{0}} u_{l}(\varepsilon) v_{s}(\varepsilon) \\
\lim _{\varepsilon \rightarrow \varepsilon_{0}} u_{k}(\varepsilon) v_{r}(\varepsilon) & \lim _{\varepsilon \rightarrow \varepsilon_{0}} u_{k}(\varepsilon) v_{s}(\varepsilon)
\end{array}\right) \neq 0 .
$$

We can rewrite the determinant above in the following way: (Because all the limits are well defined we can place the limit outside)

$$
\begin{aligned}
D & =\left(\lim _{\varepsilon \rightarrow \varepsilon_{0}} u_{l}(\varepsilon) v_{r}(\varepsilon)\right)\left(\lim _{\varepsilon \rightarrow \varepsilon_{0}} u_{k}(\varepsilon) v_{s}(\varepsilon)\right)-\left(\lim _{\varepsilon \rightarrow \varepsilon_{0}} u_{l}(\varepsilon) v_{s}(\varepsilon)\right)\left(\lim _{\varepsilon \rightarrow \varepsilon_{0}} u_{k}(\varepsilon) v_{r}(\varepsilon)\right) \\
& =\lim _{\varepsilon \rightarrow \varepsilon_{0}}\left(u_{l}(\varepsilon) v_{r}(\varepsilon) u_{k}(\varepsilon) v_{s}(\varepsilon)-u_{l}(\varepsilon) v_{s}(\varepsilon) u_{k}(\varepsilon) v_{r}(\varepsilon)\right) \\
& =\lim _{\varepsilon \rightarrow \varepsilon_{0}}(0) \\
& =0
\end{aligned}
$$

This leads to a contradiction. 
$\Leftarrow$ Suppose a semiseparable matrix $S$ is given such that it cannot be represented by two generators. Then there exists a sequence $S(u(\varepsilon), v(\varepsilon))$ with $\varepsilon \rightarrow \varepsilon_{0}$ such that

$$
\lim _{\varepsilon \rightarrow \varepsilon_{0}} S(u(\varepsilon), v(\varepsilon))=S
$$

According to Proposition 4.1 the matrix can be written as a block diagonal matrix, consisting of 2 diagonal blocks (more diagonal blocks can be dealt with in an analogous way), which can both be represented by two generators, i.e., $S$ has the following structure:

$$
S=\left(\begin{array}{cc}
S(u, v) & 0 \\
0 & S(s, t)
\end{array}\right) .
$$

In a straightforward way we can define the generators $u(\varepsilon), v(\varepsilon)$ :

$$
\begin{aligned}
& u(\varepsilon)=\left[\frac{u_{1}}{\varepsilon}, \ldots, \frac{u_{k}}{\varepsilon}, s_{1}, \ldots, s_{l}\right] \\
& v(\varepsilon)=\left[\varepsilon v_{1}, \ldots, \varepsilon v_{k}, t_{1}, \ldots, t_{n}\right] .
\end{aligned}
$$

It is clearly seen that the limit:

$$
\lim _{\varepsilon \rightarrow 0} S(u(\varepsilon), v(\varepsilon))=S .
$$

This proves the theorem.

The proof shows that the limit

$$
\lim _{\varepsilon \rightarrow 0} S(u(\varepsilon), v(\varepsilon))=S
$$

exists, but the limits of the generating vectors

$$
\begin{aligned}
& u(\varepsilon)=\left[\frac{u_{1}}{\varepsilon}, \ldots, \frac{u_{k}}{\varepsilon}, s_{1}, \ldots, s_{l}\right] \\
& v(\varepsilon)=\left[\varepsilon v_{1}, \ldots, \varepsilon v_{k}, t_{1}, \ldots, t_{n}\right]
\end{aligned}
$$

do not necessarily exist. In fact for $\varepsilon \rightarrow 0$ some elements of $u(\varepsilon)$ will become extremely large, while some elements of $v(\varepsilon)$ will become extremely small. This is the behaviour observed in Example 2.

The last theorem is an extension of Theorem 1.1.

Theorem 4.3 Suppose A is a symmetric invertible tridiagonal matrix, then its inverse is a semiseparable matrix $S$ as defined in Definition 4.

PROOF: If all the subdiagonal elements of the symmetric tridiagonal matrix are different from zero, i.e., if it is unreducible, it is proved in Theorem 1.1 that the inverse is semiseparable and representable by two generators $u$ and $v$.

Without loss of generality, we can assume that only one sub-diagonal element is different from zero (When there are more zero elements, the same arguments can be 
considered). Because one subdiagonal element equals zero, we can write the matrix $A$ in the following way:

$$
A=\left(\begin{array}{cc}
A_{1} & 0 \\
0 & A_{2}
\end{array}\right)
$$

where $A_{1}$ and $A_{2}$ are two symmetric tridiagonal matrices with nonzero subdiagonal elements, the inverses of these matrices are semiseparable matrices $S\left(u_{1}, v_{1}\right)$ and $S\left(u_{2}, v_{2}\right)$. This means that the inverse $S$ of the matrix $A$ looks like:

$$
S=\left(\begin{array}{cc}
S\left(u_{1}, v_{1}\right) & 0 \\
0 & S\left(u_{2}, v_{2}\right)
\end{array}\right)
$$

Even though this matrix cannot be represented with two generators, one can clearly see that it satisfies Definition 4. The case when there are more zero subdiagonal elements can be dealt with in the same way, because one can split the matrix in more blocks.

This last theorem proves that we have indeed a real expansion of the class of semiseparable matrices. Satisfying all the properties of the class of tridiagonal matrices, except for the representability, but this will be solved in the next section.

\section{The definition of a representation}

Before investigating different possible representations, it is necessary to define what is exactly meant when talking about a representation of a tridiagonal or a semiseparable matrix.

We define a representation based on a map as follows:

Definition 5 Suppose we have a map $r$

$$
r: \mathcal{V} \rightarrow \mathcal{U} \subseteq \mathcal{W}
$$

with the following properties $(\mathcal{V}, \mathcal{W}$ are vector spaces and $\mathcal{U}$ is a set):

- $\operatorname{dim}(\mathcal{V}) \leq \operatorname{dim}(\mathcal{W})$

- $r(\mathcal{V})=\mathcal{U}$, i.e. the map is surjective

- $\exists$ a map $s: \mathcal{U} \rightarrow \mathcal{V}$ such that $\left.r\right|_{s(\mathcal{U})}$ is bijective.

Then this map $r$ is called a representation map of the set $\mathcal{U}$. The element $v \in s(\mathcal{U}) \subseteq \mathcal{V}$ for which $r(v)=u$ with $u \in \mathcal{U}$ is called a representation of $u$.

To check if this definition suits our needs, we investigate the following map, when studying tridiagonal matrices. First we denote the class of symmetric tridiagonal matrices with:

$$
\mathcal{T}=\left\{A \in \mathbb{R}^{n \times n} \mid A \text { is a symmetric tridiagonal matrix }\right\}
$$


We have the following map:

$$
\begin{aligned}
& r_{\mathcal{T}}: \mathbb{R}^{n-1} \times \mathbb{R}^{n} \rightarrow \mathcal{T} \\
& \left(d^{(s)}, d\right) \mapsto T=\left(\begin{array}{ccccc}
d_{1} & d_{1}^{(s)} & 0 & \ldots & 0 \\
d_{1}^{(s)} & d_{2} & d_{2}^{(s)} & & \\
0 & d_{2}^{(s)} & \ddots & \ddots & \\
& \ddots & \ddots & & d_{n-1}^{(s)} \\
& & 0 & d_{n-1}^{(s)} & d_{n}
\end{array}\right)
\end{aligned}
$$

It can clearly be seen that all the properties from Definition 5 are satisfied, and the class of tridiagonal matrices $\mathcal{T}$ is clearly closed for the pointwise limit. The map $s_{\mathcal{T}}$ can be defined very easily as $s_{\mathcal{T}}=r_{\mathcal{T}}^{-1}$. This states the fact that we can use the diagonal and subdiagonal of a tridiagonal matrix to represent it.

\subsection{The representation of semiseparable matrices with generators}

Now we will work with the general class of semiseparable matrices as defined in Definition 4.

$\mathcal{S}=\left\{A \in \mathbb{R}^{n \times n} \mid A\right.$ is a symmetric semiseparable matrix of semiseparability rank 1 according to Definition 4$\}$

First of all we investigate if the map corresponding to Definition 3 satisfies the properties of a representation map. This map $r_{\mathcal{S}_{1}}$ is defined in the following natural way:

$$
\begin{aligned}
r_{\mathcal{S}_{1}}: \mathbb{R}^{n} \times \mathbb{R}^{n} & \rightarrow \mathcal{S} \\
(u, v) & \mapsto \operatorname{tril}\left(u v^{T}\right)+\operatorname{triu}\left(v u^{T}, 1\right)
\end{aligned}
$$

What one would expect here is that the definition of the representation map would reveal all the problems mentioned above, and that therefore this map cannot be a suitable representation map. Nevertheless we will try to make the map as close to a representation map as possible. The first demand on the map: $\operatorname{dim}\left(\mathbb{R}^{n} \times \mathbb{R}^{n}\right)<\operatorname{dim}\left(\mathbb{R}^{n \times n}\right)$ is satisfied in a natural way. The surjectivity condition however leads to problems. E.g. the matrix

$$
S_{1}=\left(\begin{array}{lll}
0 & 1 & 0 \\
1 & 0 & 0 \\
0 & 0 & 1
\end{array}\right)
$$

belongs to the class of semiseparable matrices $S$ but $S_{1} \notin r_{\mathcal{S}_{1}}\left(\mathbb{R}^{n} \times \mathbb{R}^{n}\right)$. Therefore this representation can never be used to represent the complete class of semiseparable matrices, so the target set needs to be adapted. Denote

$$
\mathcal{S}_{u v}=r_{\mathcal{S}_{1}}\left(\mathbb{R}^{n} \times \mathbb{R}^{n}\right)=\{A \in \mathcal{S} \mid A \text { can be represented by } u \text { and } v\}
$$

and let us restrict the map to this subclass of the semiseparable matrices. Redefine $r_{\mathcal{S}_{1}}$ as

$$
\begin{aligned}
r_{S_{1}}: \mathbb{R}^{n} \times \mathbb{R}^{n} & \rightarrow \mathcal{S}_{u v} \\
(u, v) & \mapsto \operatorname{tril}\left(u v^{T}\right)+\operatorname{triu}\left(v u^{T}, 1\right)
\end{aligned}
$$


Perhaps this restriction will make $r_{\mathcal{S}_{1}}$ a good representation for the class $\mathcal{S}_{u v}$. The surjectivity is already satisfied. We will now search for the function $s_{\mathcal{S}_{1}}$. It is clearly seen that the inverse of $r_{S_{1}}$ will not suit our needs, as shown by the following example:

Example 3 Take the following two vectors: $u_{1}=[1,2,3]^{T}$ and $v_{1}=[2,2,2]^{T}$, the matrix $r_{S_{1}}\left(u_{1}, v_{1}\right)$ is the following:

$$
r_{S_{1}}\left(u_{1}, v_{2}\right)=\left(\begin{array}{lll}
2 & 4 & 6 \\
4 & 4 & 6 \\
6 & 6 & 6
\end{array}\right) .
$$

Constructing $r_{S_{1}}\left(u_{2}, v_{2}\right)$ with $u_{2}=[2,4,6]^{T}$ and $v_{2}=[1,1,1]^{T}$ gives the following result:

$$
r_{S_{1}}\left(u_{2}, v_{2}\right)=\left(\begin{array}{lll}
2 & 4 & 6 \\
4 & 4 & 6 \\
6 & 6 & 6
\end{array}\right) \text {. }
$$

This means that the map

$$
\begin{aligned}
r_{\mathcal{S}_{1}}: r_{\mathcal{S}_{1}}^{-1}\left(\mathcal{S}_{u v}\right)=\mathbb{R}^{n} \times \mathbb{R}^{n} & \rightarrow S_{u v} \\
(u, v) & \mapsto \operatorname{tril}\left(u v^{T}\right)+\operatorname{triu}\left(v u^{T}, 1\right)
\end{aligned}
$$

can never be bijective.

Some kind of normalization is needed such that every matrix will have a unique set of generators. The demand $u_{1}=1$ will often work for a unique representation, but not always.

Example 4 Suppose the following matrix $S$ is given:

$$
\left(\begin{array}{lll}
0 & 0 & 0 \\
0 & 2 & 3 \\
0 & 3 & 6
\end{array}\right)
$$

The following vectors both have the first element of the vector u equal to 1 but are not the same: $u_{1}=[1,2,3]^{T}, v_{1}=[0,1,2]^{T}$ and the couple $u_{1}=[1,1,3 / 2]^{T}, v_{1}=[0,2,4]^{T}$.

Therefore $s_{\mathcal{S}_{1}}$ needs to be defined in a solid way. Suppose we have a matrix $S \in S_{u v}$, $S=\left[K_{1}, K_{2}, \ldots, K_{n}\right]$, when the $K_{i}$ 's denote the columns of the matrix $S$. Suppose $1 \leq$ $l \leq k \leq n$ such that $K_{l}$ is the first column in $S$ different from zero, and $K_{k}$ is the last column in $S$ different from zero. Define then $u=K_{l} / S(k, l)$ and $v=K_{k}$, then we get a unique set $(u, v)$ for every semiseparable matrix in $S_{u v}$. Using these calculations as a standard definition for $u$ and $v$ we can define the function $s_{\mathcal{S}_{1}}$ in the following way:

Definition 6 Suppose for each matrix $S \in \mathcal{S}_{u v}$ of dimension $n, K_{l}$ is the first column of $S$ different from zero and $K_{k}$ is the last column of $S$ different from zero. Then we define the map $s_{\mathcal{S}_{1}}$ in the following way:

$$
\begin{aligned}
s_{\mathcal{S}_{1}}: \mathcal{S}_{u v} & \rightarrow \mathbb{R}^{n} \times \mathbb{R}^{n} \\
S & \mapsto\left(\frac{K_{l}}{S(k, l)}, K_{k}\right)
\end{aligned}
$$


Because this defines the projection into the vectors $u$ and $v$ in a unique way, we have that $\left.r_{S_{1}}\right|_{s\left(\mathcal{S}_{u v}\right)}$ is bijective. We have now a unique representation for each element of the set $\mathcal{S}_{u v}$ but we want to represent the complete set $\mathcal{S}$. To represent the complete class $\mathcal{S}$ and to overcome the numerical instabilities we have to search for a new representation.

\subsection{A new representation for semiseparable matrices}

We consider a new type of representation. For a semiseparable matrix of dimension $n$, this representation consists of $n-1$ Givens transformations and a vector of length $n$. The Givens transformations are denoted as $G=\left[G_{1}, \ldots, G_{n-1}\right]$ and the vector as $d=\left[d_{1}, \ldots, d_{n}\right]$. It is clearly seen that this representation also keeps $O(n)$ information to reconstruct the complete semiseparable matrix.

The following figures denote how the semiseparable matrix can be reconstructed, using this information. The elements denoted by $\nabla$ make up the semiseparable part of the matrix. Initially one starts on the first 2 rows of the matrix. The element $d_{1}$ is placed in the upper left position, then a Givens transformation is applied, and finally to complete the first step element $d_{2}$ is added in position $(2,1)$. Only the first two columns and rows are shown here.

$$
\left(\begin{array}{cc}
d_{1} & 0 \\
0 & 0
\end{array}\right) \rightarrow G_{1}\left(\begin{array}{cc}
d_{1} & 0 \\
0 & 0
\end{array}\right)+\left(\begin{array}{cc}
0 & 0 \\
0 & d_{2}
\end{array}\right) \rightarrow\left(\begin{array}{cc}
\bigotimes & 0 \\
\square & d_{2}
\end{array}\right)
$$

The second step consists of applying the Givens transformation $G_{2}$ on the second and the third row, furthermore $d_{3}$ is added in position $(3,3)$. Here only the first three columns are shown and the second and third row. This leads to:

$$
\left(\begin{array}{ccc}
\otimes & d_{2} & 0 \\
0 & 0 & 0
\end{array}\right) \rightarrow G_{2}\left(\begin{array}{ccc}
\otimes & d_{2} & 0 \\
0 & 0 & 0
\end{array}\right)+\left(\begin{array}{ccc}
0 & 0 & 0 \\
0 & 0 & d_{3}
\end{array}\right) \rightarrow\left(\begin{array}{ccc}
\otimes & \bigotimes & 0 \\
\square & \bigotimes & d_{3}
\end{array}\right)
$$

This process can be repeated by applying the Givens transformation $G_{3}$ on the third and the fourth row of the matrix, and afterwards adding the diagonal element $d_{4}$. After applying all the Givens transformations and adding all the diagonal elements, the lower triangular part of a symmetric semiseparable matrix is constructed. Because of the symmetry also the upper triangular part is known.

Suppose the Givens and vector representation of a semiseparable matrix $S$ is known. When denoting the Givens transformations as:

$$
G_{l}=\left(\begin{array}{rr}
c_{l} & -s_{l} \\
s_{l} & c_{l}
\end{array}\right)
$$

The elements $S(i, j)$ with $n>i \geq j$ are calculated in the following way:

$S(i, j)=c_{i} s_{i-1} s_{i-2} \cdots s_{j} d_{j}$. When $n=i$ we have $S(i, j)=s_{n-1} s_{n-2} \cdots s_{j} d_{j}$. When $n \geq$ $j>i, S(i, j)$ can be calculated in a similar way, because of the symmetry. The elements of the semiseparable matrix can therefore be calculated in a stable way based on the Givens vector representation. This means that we have constructed the following map 
$r_{S_{2}}$

$$
\begin{aligned}
r_{S_{2}}: \mathbb{R}^{2 \times(n-1)} \times \mathbb{R}^{n} & \rightarrow \mathbb{R}^{n \times n} \\
{\left[\left(\begin{array}{ccc}
c_{1} & \ldots & c_{n-1} \\
s_{1} & \ldots & s_{n-1}
\end{array}\right),\left(d_{1}, \ldots, d_{n}\right)\right] } & \mapsto\left(\begin{array}{cccc}
c_{1} d_{1} & & & \\
c_{2} s_{1} d_{1} & c_{2} d_{2} & & \\
c_{3} s_{2} s_{1} d_{1} & c_{3} s_{2} d_{2} & c_{3} d_{3} & \\
\vdots & \vdots & & \ddots
\end{array}\right)
\end{aligned}
$$

Example 5 (Example 1 Continued) The Givens-vector representation of the matrix in Example 2 is the following: (In the first row of $G$ the elements $c_{1}, \ldots, c_{4}$ are stored and in the second row the elements $s_{1}, \ldots, s_{4}$.)

$$
G=\left(\begin{array}{rrrl}
9.0903 \cdot 10^{-1} & 9.7620 \cdot 10^{-1} & 9.9999 \cdot 10^{-1} & 1.0000 \\
-4.1672 \cdot 10^{-1} & -2.1686 \cdot 10^{-1} & -1.2997 \cdot 10^{-3} & 4.8030 \cdot 10^{-10}
\end{array}\right)
$$

and

$$
d=\left(\begin{array}{lllll}
1.4012 & 2.2778 & 2.5026 & 1.0000 \cdot 10^{2} & 1.0000 \cdot 10^{5}
\end{array}\right)
$$

All the elements of the semiseparable matrix can be reconstructed now with high relative precision if the corresponding elements of $G$ and $d$ are known with high relative precision.

\subsection{Retrieving the representation from a semiseparable matrix}

Here, a method is proposed to retrieve the representation of a semiseparable matrix, in terms of the Givens-vector representation in a stable way.

In fact we search for the map:

$$
\begin{aligned}
s_{\mathcal{S}_{2}}: S & \rightarrow \mathbb{R}^{2 \times(n-1)} \times \mathbb{R}^{n} \\
S & \mapsto(G, d)
\end{aligned}
$$

Suppose we have a semiseparable matrix as in (17). The vector elements $d_{i}$ can be retrieved rather easily, from the matrix. In fact:

$$
\begin{aligned}
& \|S(i: n, i)\|_{2} \\
= & \sqrt{\left(c_{i} d_{i}\right)^{2}+\left(c_{i+1} s_{i} d_{i}\right)^{2}+\cdots+\left(c_{n-1} s_{n-2} \ldots s_{i} d_{i}\right)^{2}+\left(s_{n-1} s_{n-2} \ldots s_{i} d_{i}\right)^{2}} \\
= & \sqrt{\left(c_{i} d_{i}\right)^{2}+\left(c_{i+1} s_{i} d_{i}\right)^{2}+\cdots+\left(s_{n-1}^{2}+c_{n-1}^{2}\right)\left(s_{n-2} \ldots s_{i} d_{i}\right)^{2}} \\
= & \sqrt{\left(c_{i}^{2}+s_{i}^{2}\right) d_{i}^{2}} \\
= & \left|d_{i}\right|
\end{aligned}
$$

This means that the absolute values of $d_{i}$ can be calculated by calculating the norms of the $i$ th column $\|S(i: n, i)\|$. To calculate the corresponding Givens transformations connected with the semiseparable matrix, we first map the matrix $S$ towards another semiseparable matrix. This procedure is quite expensive, but results in a stable way to 
compute a representation. The matrix $S$ is mapped onto the following matrix of norms. Note that the choice of the norm does not play a role:

$$
\hat{S}=\left(\begin{array}{ccc}
\|S(1,1)\| & & \\
\|S(2,1)\| & \|S(2,1: 2)\| & \\
\vdots & & \ddots \\
\|S(n, 1)\| & \|S(n, 1: 2)\| & \ldots\|S(n, 1: n)\|
\end{array}\right)
$$

So in fact a new matrix $\hat{S}$ is created with as elements $\hat{S}_{i, j}=\|S(i, 1: j)\|$. Remark that this matrix has the same dependency between the rows as the matrix $S$ (except for the signs). We start with calculating the last Givens transformation $G_{n-1}$ such that

$$
G_{n-1}\left(r_{n-1}, 0\right)^{T}=(\|S(n-1,1: n-1)\|,\|S(n, 1: n-1)\|)^{T} .
$$

Before calculating the next Givens transformation we have to update the matrix $\hat{S}$, by applying the Givens transformation $G_{n-2}$ to the rows $n-1$ and $n$. Denoting this new matrix as $\hat{S}^{(n-1)}$, the next Givens transformation $G_{n-2}$ is calculated such that :

$$
G_{n-2}\left(r_{n-2}, 0\right)^{T}=\left(\|S(n-2,1: n-2)\|, \hat{S}_{n-2, n-2}^{(n-2)}\right)^{T} .
$$

Updating again the matrix $\hat{S}^{(n-2)}$ by applying the Givens transformation $G_{n-2}^{T}$ to the rows $n-2$ and $n-1$ we get the matrix $\hat{S}^{(n-3)}$ and we can calculate $G_{n-3}$. Consequetively, all the Givens transformations can be calculated, satisfying:

$$
G_{i}\left(r_{i}, 0\right)^{T}=\left(\|S(i, 1: i)\|, \hat{S}_{i, i}^{(i)}\right)^{T} .
$$

This procedure gives us the Givens-vector representation, except for the sign, but this is done rather easily, by taking a look at the signs of the original matrix, because the Givens are uniquely determined, because we take $c_{i}$ always positive. And when a Givens transformation of the following form has to be determined: $G(0,0)^{T}=(0,0)$ we take $G$ equal to the identity matrix.

This reconstruction of the representation is of course quite expensive, (faster, even $O(n)$ algorithms, can be constructed, for arbitrary semiseparable matrices, but they are numerically unstable). In practice however, one should take a closer look at the application one is working with, probably much faster algorithms can be devised corresponding to a particular problem.

In the reduction algorithm from symmetric to semiseparable, the output of the algorithms is already in the appropriate form [41], also the $Q R$ algorithm as proposed in [43] uses as input this representation and gives as output the Givens vector representation of the new semiseparable matrix. In both of these algorithms there is no need to apply this expensive procedure for calculating the Givens vector representation.

This last procedure justifies our new representation, because the mappings $r_{\mathcal{S}_{2}}$ and $s_{\mathcal{S}_{2}}$ are defined in the appropriate way and represent the complete set $S$.

Moreover, this representation reveals already the $Q R$ factorization of the semiseparable matrix $S$ : the Givens transformations appearing in the representation of the matrix are exactly the same as the Givens transformations appearing in the $Q$ factor of the $Q R$ factorization.(More information can be found in [42, 34].) 


\section{An algorithm connected to the representation}

In this section one example of an implementation with the new definition is given. In $[42,44]$ other algorithms, based on the Givens-vector representation can be found. The algorithms for retrieving the representation and for constructing the full semiseparable matrix, given the Givens-vector representation, can be deduced easily with the information in the previous section.

Here an $O(n)$ implementation of a matrix vector multiplication will be given. The formulas will be given for nonsymmetric semiseparable matrices, who have two sequences of Givens transformations and two vectors. Let us denote the first sequence as:

$$
\begin{aligned}
G & =\left(\begin{array}{llll}
c_{1} & c_{2} & \ldots & c_{n-1} \\
s_{1} & s_{2} & \ldots & s_{n-1}
\end{array}\right) \\
d & =\left(\begin{array}{llll}
d_{1} & d_{2} & \ldots & d_{n}
\end{array}\right)
\end{aligned}
$$

and the second as:

$$
\begin{aligned}
H & =\left(\begin{array}{llll}
r_{1} & r_{2} & \ldots & r_{n-2} \\
t_{1} & t_{2} & \ldots & t_{n-2}
\end{array}\right) \\
e & =\left(\begin{array}{llll}
e_{1} & e_{2} & \ldots & e_{n-1}
\end{array}\right) .
\end{aligned}
$$

We want to calculate the multiplication between $S$ and $v$ where

$$
S=\left(\begin{array}{cccccc}
c_{1} d_{1} & r_{1} e_{1} & r_{2} t_{1} e_{1} & \cdots & r_{n-2} t_{n-3} \cdots t_{1} e_{1} & t_{n-2} t_{n-3} \cdots t_{1} e_{1} \\
c_{2} s_{1} d_{1} & c_{2} d_{2} & r_{2} e_{2} & & \vdots & \vdots \\
c_{3} s_{2} s_{1} d_{1} & c_{3} s_{2} d_{2} & c_{3} d_{3} & \ddots & & \\
\vdots & & \ddots & \ddots & r_{n-2} e_{n-2} & t_{n-2} e_{n-2} \\
c_{n-1} s_{n-2} \cdots s_{1} d_{1} & \ldots & & & c_{n-1} d_{n-1} & e_{n-1} \\
s_{n-1} s_{n-2} \cdots s_{1} d_{1} & \cdots & & & s_{n-1} d_{n-1} & d_{n}
\end{array}\right)
$$

and

$$
v=\left(\begin{array}{llll}
v_{1} & v_{2} & \ldots & v_{n}
\end{array}\right)^{T} .
$$

To deduce the algorithm, we have to decompose the matrix into a strict uppertriangular, and a lower triangular part of $S$. Denote $S_{1}=\operatorname{tril}(S)$ and $S_{2}=\operatorname{triu}(S)$. We will now compute:

$$
\begin{aligned}
& y=S_{1} v \\
& z=S_{2} v
\end{aligned}
$$

such that $x=y+z=S v$ is the solution. To calculate $y=\left(\begin{array}{cccc}y_{1} & y_{2} & \ldots & y_{n}\end{array}\right)$ in a fast way, we rewrite the following formulas (only the first 4 components of $y$ are denoted):

$$
\begin{aligned}
& y_{1}=c_{1} d_{1} v_{1} \\
& y_{2}=c_{2} s_{1} d_{1} v_{1}+c_{2} d_{2} v_{2} \\
& y_{3}=c_{3} s_{2} s_{1} d_{1} v_{1}+c_{3} s_{2} d_{2} v_{2}+c_{3} d_{3} v_{3} \\
& y_{4}=c_{4} s_{3} s_{2} s_{1} d_{1} v_{1}+c_{4} s_{3} s_{2} d_{2} v_{2}+c_{4} s_{3} d_{3} v_{3}+c_{4} d_{4} v_{4}
\end{aligned}
$$


We use some help variables called $a_{i}$. Rewriting the formulas reveals the order $O(n)$ algorithm for the multiplication.

$$
\begin{aligned}
y_{1} & =c_{1}\left(d_{1} v_{1}\right) \\
& =c_{1} a_{1} \\
y_{2} & =c_{2}\left(s_{1} d_{1} v_{1}+d_{2} v_{2}\right) \\
& =c_{2}\left(s_{1} a_{1}+d_{2} v_{2}\right) \\
& =c_{2} a_{2} \\
y_{3} & =c_{3}\left(s_{2}\left(s_{1} d_{1} v_{1}+d_{2} v_{2}\right)+d_{3} v_{3}\right) \\
& =c_{3}\left(s_{2} a_{2}+d_{3} v_{3}\right) \\
& =c_{3} a_{3} \\
y_{4} & =c_{4}\left(s_{3}\left(s_{2}\left(s_{1} d_{1} v_{1}+d_{2} v_{2}\right)+d_{3} v_{3}\right) v_{3}+d_{4} v_{4}\right) \\
& =c_{4}\left(s_{3} a_{3}+d_{4} v_{4}\right) \\
& =c_{4} a_{4} .
\end{aligned}
$$

Combining the last 2 equalities of all the $y_{i}$ one can derive an order $n$ algorithm to perform the multiplication of $S_{1}$ and $v$. The multiplication of $S_{2}$ and $v$ can be derived in a completely analogous way.

\section{Conclusions}

In this paper, we have shown that the standard definition and representation of semiseparable matrices based on generators has some disadvantages. We have given an alternative definition and a corresponding representation which keeps the interesting properties of the standard representation but does not exhibit the disadvantages.

\section{Appendix A}

Our main result here will be to prove, as in [21, Chapter II], that the inverse of a one-pair matrix is an unreducible tridiagonal matrix and vice versa.

First some notation has to be introduced. Suppose we have an arbitrary $n \times n$ matrix $A$ which is denoted as $A=\left(a_{i, j}\right)_{i, j \in\{1, \ldots, n\}}$

Definition 7 Define the matrix $A\left(i_{1}, \ldots, i_{p} ; j_{1}, \ldots, j_{p}\right)$ as the matrix:

$$
A\left(i_{1}, \ldots, i_{p} ; j_{1}, \ldots, j_{p}\right)=\left(a_{i, j}\right)
$$

with the indices belonging to the following sets:

$$
\begin{gathered}
i \in\left\{i_{1}, \ldots, i_{p}\right\} \\
j \in\left\{j_{1}, \ldots, j_{p}\right\} .
\end{gathered}
$$

For the determinant of a matrix we will use the following notation:

$$
\operatorname{det}(A)=|A|
$$


Before we can prove the first important statement a proposition is needed.

Proposition 7.1 Suppose a Jacobi matrix (this is a tridiagonal matrix) $J$ of size $n$ is given. If

$$
\begin{gathered}
1 \leq i_{1}<i_{2}<\ldots<i_{p} \leq n \\
1 \leq j_{1}<j_{2}<\ldots<j_{p} \leq n
\end{gathered}
$$

and

$$
\begin{aligned}
& i_{1}=j_{1}, i_{2}=j_{2}, \ldots, i_{v_{1}}=j_{v_{1}}, \\
& i_{v_{1}+1} \neq j_{v_{1}+1}, \ldots, i_{v_{2}} \neq j_{v_{2}}, \\
& i_{v_{2}+1}=j_{v_{2}+1}, \ldots, i_{v_{3}}=j_{v_{3}}, \\
& i_{v_{3}+1} \neq j_{v_{3}+1}, \ldots
\end{aligned}
$$

then

$$
\begin{aligned}
& \left|J\left(i_{1}, \ldots, i_{p} ; j_{1}, \ldots, j_{p}\right)\right| \\
= & \left|J\left(i_{1}, \ldots, i_{v_{1}} ; j_{1}, \ldots, j_{v_{1}}\right)\right| \cdot\left|J\left(i_{v_{1}+1} ; j_{v_{1}+1}\right)\right| \ldots \\
& \left|J\left(i_{v_{2}} ; j_{v_{2}}\right)\right| \cdot\left|J\left(i_{v_{2}+1}, \ldots, i_{v_{3}} ; j_{v_{2}+1}, \ldots, j_{v_{3}}\right)\right| \ldots
\end{aligned}
$$

PROOF: In fact it is enough to prove that under the conditions (20), and $i_{v} \neq j_{v}$ the following equations hold:

$$
\begin{aligned}
& \left|J\left(i_{1}, \ldots, i_{p} ; j_{1}, \ldots, j_{p}\right)\right| \\
= & \left|J\left(i_{1}, \ldots, i_{v} ; j_{1}, \ldots, j_{v}\right)\right| \cdot\left|J\left(i_{v+1}, \ldots, i_{p} ; j_{v+1}, \ldots, j_{p}\right)\right| \\
= & \left|J\left(i_{1}, \ldots, i_{v-1} ; j_{1}, \ldots, j_{v-1}\right)\right| \cdot\left|J\left(i_{v}, \ldots, i_{p} ; j_{v}, \ldots, j_{p}\right)\right|
\end{aligned}
$$

Proving the first of the two equations is enough. If $i_{v}<j_{v}$ then we have, because the matrix $A$ is tridiagonal:

$$
a_{i_{\lambda} j_{\mu}}=0 \quad(\lambda=1,2, \ldots, v ; \mu=v+1, \ldots, p)
$$

otherwise $i_{v}>j_{v}$ would lead to:

$$
a_{i_{\lambda} j_{\mu}}=0 \quad(\lambda=v+1, \ldots, p ; \mu=1,2, \ldots, v)
$$

These two last statements say that the matrix is either upper block triangular, or lower block triangular. This proves (21).

Now it is time to prove that the inverse of a symmetric Jacobi matrix is a so called one-pair matrix.

PROOF: of Theorem 1.1 We will prove the theorem by explicitely constructing the inverse of the tridiagonal matrix $A$. This matrix will then appear to be a one-pair matrix. Suppose $S$ is the inverse of the matrix $A$, this means that:

$$
S(i, j)=\frac{1}{\operatorname{det}(A)}(-1)^{i+j}|A(1, \ldots, i-1, i+1, \ldots, n ; 1, \ldots, j-1, j+1, \ldots, n)|
$$

We distinguish between two cases now: 
1. When $i \leq j$

$$
\begin{aligned}
S(i, j)= & \frac{1}{\operatorname{det}(A)}(-1)^{i+j}|A(1, \ldots, i-1 ; 1, \ldots, i-1)| \cdot|A(i, i+1)| \cdots \\
& |A(j-1, j)| \cdot|A(j+1, \ldots, n ; j+1, \ldots, n)| \\
= & \frac{1}{\operatorname{det}(A)}(-1)^{i+j}|A(1, \ldots, i-1 ; 1, \ldots, i-1)| b_{i} b_{i+1} \ldots \\
& b_{j-1}|A(j+1, \ldots, n ; j+1, \ldots, n)|
\end{aligned}
$$

2. When $i \geq j$, we can do the same as above and one gets

$$
\begin{aligned}
S(i, j)= & \frac{1}{\operatorname{det}(A)}(-1)^{i+j}|A(1, \ldots, j-1 ; 1, \ldots, j-1)| b_{j} b_{j+1} \ldots \\
& b_{i-1}|A(i+1, \ldots, n ; i+1, \ldots, n)|
\end{aligned}
$$

When writing $u_{i}$ and $v_{j}$ now as: (Under the assumption that all the $b_{i} \neq 0$ )

$$
\begin{aligned}
u_{i} & =\frac{(-1)^{i}}{\operatorname{det}(A)}|A(1, \ldots, i-1 ; 1, \ldots, i-1)| b_{i} b_{i}+1 \ldots b_{n-1} \\
v_{i} & =\frac{(-1)^{i}|A(1, \ldots, i-1 ; 1, \ldots, i-1)|}{b_{i} b_{i}+1 \ldots b_{n-1}}
\end{aligned}
$$

This means that

$$
S(i, j)= \begin{cases}u_{i} v_{j} & (i \leq j) \\ u_{j} v_{i} & (i \geq j)\end{cases}
$$

This proves the theorem.

Proposition 7.2 Suppose we have a one-pair matrix $S$ which is generated by the vectors $u$ and $v$. If

$$
1 \leq i_{1}, j_{1}<i_{2}, j_{2}<\ldots<i_{p}, j_{p} \leq n
$$

then

$$
S\left(i_{1}, \ldots, i_{p} ; j_{1}, \ldots, j_{p}\right)=u_{\alpha_{1}}\left|\begin{array}{ll}
v_{\beta_{1}} & v_{\alpha_{2}} \\
u_{\beta_{1}} & u_{\alpha_{2}}
\end{array}\right|\left|\begin{array}{cc}
v_{\beta_{2}} & v_{\alpha_{3}} \\
u_{\beta_{2}} & u_{\alpha_{3}}
\end{array}\right| \ldots\left|\begin{array}{cc}
v_{\beta_{p-1}} & v_{\alpha_{p}} \\
u_{\beta_{p-1}} & u_{\alpha_{p}}
\end{array}\right| v_{\beta_{p}}
$$

where

$$
\alpha_{v}=\min \left(i_{v}, j_{v}\right) \quad \beta_{v}=\max \left(i_{v}, j_{v}\right)
$$

PROOF: Because the matrix $S$ is a symmetric matrix, we can without loss of generality assume that $i_{2} \leq j_{2}$, this means that $\alpha_{2}=i_{2}$ and $\beta_{2}=j_{2}$, leading to the following equality:

$$
\begin{aligned}
& \left|S\left(i_{1}, \ldots, i_{p} ; j_{1}, \ldots, j_{p}\right)\right| \\
= & \operatorname{det}\left(\begin{array}{ccccc}
u_{\alpha_{1}} v_{\beta_{1}} & u_{i_{1}} v_{j_{2}} & u_{i_{1}} v_{j_{3}} & \ldots & u_{i_{1}} v_{j_{p}} \\
u_{j_{1}} v_{i_{2}} & u_{i_{2}} v_{j_{2}} & u_{i_{2}} v_{j_{3}} & \ldots & u_{i_{2}} v_{j_{p}} \\
\vdots & & \ddots &
\end{array}\right) .
\end{aligned}
$$


Subtracting from the first row the second one multiplied with $u_{i_{1}} / v_{i_{2}}$ gives the following equation:

$$
\begin{aligned}
& \left|S\left(i_{1}, \ldots, i_{p} ; j_{1}, \ldots, j_{p}\right)\right| \\
= & \left(u_{\alpha_{1}} v_{\beta_{1}}-\frac{u_{k_{1}} v_{i_{2}} u_{i_{1}}}{u_{i_{2}}}\right)\left|S\left(i_{2}, \ldots, i_{p} ; j_{2}, \ldots, j_{p}\right)\right| \\
= & \frac{u_{\alpha_{1}}}{u_{\alpha_{2}}}\left|\begin{array}{cc}
v_{\beta_{1}} & v_{\alpha_{2}} \\
u_{\beta_{1}} & u_{\alpha_{2}}
\end{array}\right|\left|S\left(i_{2}, \ldots, i_{p} ; j_{2}, \ldots, j_{p}\right)\right|
\end{aligned}
$$

Applying the equation above successively and using the fact that

$$
S\left(i_{p} ; j_{p}\right)=u_{\alpha_{p}} v_{\alpha_{p}}
$$

gives the desired result.

One more proposition about the determinants of minors of a one-pair matrix is needed

Proposition 7.3 Suppose we have a one-pair matrix $S$. If

$$
\begin{array}{r}
1 \leq i_{1}<i_{2}<\ldots<i_{p} \leq n \\
1 \leq j_{1}<j_{2}<\ldots<j_{p}
\end{array}
$$

but equation (25) is not satisfied then:

$$
\left|S\left(i_{1}, \ldots, i_{p} ; j_{1}, \ldots, j_{p}\right)\right|=0
$$

PROOF: Using the proposition 7.2 and assuming that

$$
1 \leq i_{1}, j_{1}<i_{2}, j_{2}<\ldots<i_{r}, j_{r}
$$

whereas for example $j_{r}>i_{r+1}$. This means that

$$
\begin{aligned}
& \left|S\left(i_{1}, \ldots, i_{p} ; j_{1}, \ldots, j_{p}\right)\right| \\
= & \frac{u_{\alpha_{1}}}{u_{\alpha_{2}}}\left|\begin{array}{ll}
v_{\beta_{1}} & v_{\alpha_{2}} \\
u_{\beta_{1}} & u_{\alpha_{2}}
\end{array}\right| \ldots\left|\begin{array}{cc}
v_{\beta_{r-1}} & v_{\alpha_{r}} \\
u_{\beta_{1-1}} & u_{\alpha_{r}}
\end{array}\right|\left|S\left(i_{r}, \ldots, i_{p} ; j_{r}, \ldots, j_{p}\right)\right| .
\end{aligned}
$$

Because $j_{r}>i_{r+1}$, this means that the last determinant is zero.

Now the theorem stating that the inverse of a one-pair matrix is a tridiagonal can be stated.

Theorem 7.4 Suppose $S$ is a one-pair matrix with all the elements of the generators different from zero, then the inverse of $S$ is a strict tridiagonal matrix.

PROOF: It is easy proved by using Propositions 7.2 and 7.3. 


\section{References}

[1] E. Asplund. Inverses of matrices $a_{i j}$ which satisfy $a_{i j}=0$ for $j>i+p$. Math. Scand, 7:57-60, 1959.

[2] W.W. Barrett and P.J. Feinsilver. Inverses of banded matrices. Linear Algebra and Its Applications, 41:111-130, 1981.

[3] S. Chandrasekaran, P. Dewilde, M. Gu, T. Pals, X. Sun, A.-J. van der Veen, and D. White. Fast stable solvers for sequentially semi-separable linear systems of equations and least squares problems. http://cobalt.et.tudelft.nl/ allejan/publications.html, preprint, 2001.

[4] S. Chandrasekaran, P. Dewilde, M. Gu, T. Pals, and A.-J. van der Veen. Fast stable solver for sequentially seni-separable linear systems of equations. Lecture Notes in Computer Science, 2552:545-554, 2002.

[5] S. Chandrasekaran and M. Gu. Fast and stable eigendecomposition of symmetric banded plus semi-separable matrices. Linear Algebra and Its Applications, 313:107-114, 2000.

[6] S. Chandrasekaran and M. Gu. A fast and stable solver for recursively semiseparable systems of linear equations. Contemporary Mathematics, 281:39-53, 2001.

[7] S. Chandrasekaran and M. Gu. A divide and conquer algorithm for the eigendecomposition of symmetric block-diagonal plus semi-separable matrices. $\mathrm{Nu}$ merische Mathematik, 2003. http://link.springer.de/link/service/journals/00211/.

[8] S. Chandrasekaran and M. Gu. Fast and stable algorithms for banded plus semiseparable systems of linear equations. SIAM J. Matrix Anal. Appl., 25(2):373-384, 2003. http://citeseer.nj.nec.com/241657.html.

[9] D. Colton and R. Kress. Inverse acoustic and electromagnetic scattering theory. Springer-Verlag, second edition, 1998.

[10] P. Dewilde and A.-J. van der Veen. Time-varying systems and computations. Kluwer academic publishers, Boston, June 1998.

[11] Y. Eidelman and I. Gohberg. Inversion formulas and linear complexity algorithm for diagonal plus semiseparable matrices. Computers \& Mathematics with Applications, 33(4):69-79, August 1996.

[12] Y. Eidelman and I. Gohberg. A look ahead block schur algorithm for diagonal plus semiseparable matrices. Computers \& Mathematics with Applications, 35(10):25-34, 1997.

[13] L. Elsner. Some observations on inverses of band matrices and low rank perturbations of triangular matrices. http://citeseer.nj.nec.com/elsner00some.html. 
[14] S.M. Fallat, M. Fiedler, and T.L. Markham. Generalized oscillatory matrices. Linear Algebra and Its Applications, 359:79-90, 2003.

[15] D. Fasino and L. Gemignani. Direct and inverse eigenvalue problems, for diagonal-plus-semiseparable matrices. Submitted for publication, 2001. http://www.dimi.uniud.it/ fasino/papers.html.

[16] D. Fasino and L. Gemignani. Structural and computational properties of possibly singular semiseparable matrices. Linear Algebra and its Applications, 340:183$198,2001$.

[17] D. Fasino, N. Mastronardi, and M. Van Barel. Fast and stable algorithms for reducing diagonal plus semi separable matrices to tridiagonal and bidiagonal form. Contemporary Mathematics, 323:105-118, 2003.

[18] M. Fiedler. Structure ranks of matrices. Linear Algebra and Its Applications, 179:119-127, 1993.

[19] M. Fiedler. Basic matrices. Linear Algebra and Its Applications, 373:143-151, 2003.

[20] M. Fiedler and Z. Vavř́n. Generalized Hessenberg matrices. Submitted for publication.

[21] F.P. Gantmacher and M.G. Krein. Oscillation matrices and kernels and small vibrations of mechanical systems. AMS Chelsea publishing, 2002.

[22] L. Gemignani and D. Fasino. Fast and stable solution of banded-plussemiseparable linear systems. Calcolo, 39(4):201-217, 2002.

[23] I. Gohberg and M.A. Kaashoek. Time varying linear systems with boundary conditions and integral operators, I. the transfer operator and its properties. Integral Equations and Operator Theory, 7:325-391, 1984.

[24] R.A. Gonzales, J. Eisert, I. Koltracht, M. Neumann, and G. Rawitscher. Integral equation method for the continuous spectrum radial schrodinger equation. Journal of computational physics, 134:134-149, 1997.

[25] F.A. Graybill. Matrices with applications in statistics. Wadsworth international group, Belmont, California, 1983.

[26] L. Greengard and V. Rokhlin. On the numerical solution of two-point boundary value problems. Communications on Pure and Applied Mathematics, 44:419452, 1991.

[27] G.J. Groenewald, M.A. Petersen, and A.C.M. Ran. Factorization of integral operators with semiseparable kernel and symmetries. http://www.cs.vu.nl/ ran/intopfac2.ps. 
[28] S.-Y. Kang, I. Koltracht, and G. Rawitscher. High accuracy method for integral equations with discontinuous kernels. http://arxiv.org/abs/math.NA/9909006, Submitted for publication.

[29] A. Kavcic and M.F. Moura. Matrices with banded inverses: inversion algorithms and factorization of gauss-markov processes. IEEE Transactions on Information Theory, 46(4):1495-1509, July 2000.

[30] J-Y. Lee and L. Greengard. A fast adaptive numerical method for stiff two-point boundary value problems. SIAM Journal on Scientific Computing, 18(2):403429, March 1997.

[31] N. Mastronardi, S. Chandrasekaran, and S. Van Huffel. Fast and stable two-way algorithm for diagonal plus semi-separable systems of linear equations. Numerical Linear Algebra with Applications, 8(1):7-12, 2001.

[32] N. Mastronardi, S. Chandrasekaran, and S. Van Huffel. Fast and stable algorithms for reducing diagonal plus semiseparable matrices to tridiagonal and bidiagonal form. BIT, 41(1):149-157, 2003.

[33] N. Mastronardi, M. Van Barel, R. Vandebril, and L. Elden. A Lanczos subspace iteration method for the symmetric rank revealing factorization. In preparation, 2003.

[34] N. Mastronardi, E. Van Camp, and M. Van Barel. Divide and conquer type algorithms for computing the eigendecomposition of diagonal plus semiseparable matrices. Technical Report 7 (5/2003), Istituto per le Applicazioni del Calcolo “M. Picone”, Consiglio Nazionale delle Ricerche, Rome, Italy, 2003.

[35] P. Rozsa. Band matrices and semi-separable matrices. Colloquia Mathematica Societatis Janos Bolyai, 50:229-237, 1986.

[36] P. Rozsa. On the inverse of band matrices. Integral Equations and Operator Theory, 10:82-95, 1987.

[37] P. Rozsa, R. Bevilacqua, F. Romani, and P. Favati. On band matrices and their inverses. Linear Algebra and Its Applications, 150:287-295, 1991.

[38] P. Rozsa and F. Romani. On periodic block tridiagonal matrices. Linear Algebra and Its Applications, 167:35-52, 1992.

[39] H.P. Jr. Starr. On the numerical solution of one-dimensional integral and differential equations. PhD thesis, Yale University, 1992. Research Report YALEU/DCS/RR-888.

[40] M. Van Barel, D. Fasino, L. Gemignani, and N. Mastronardi. Orthogonal rational functions and diagonal plus semiseparable matrices. In F.T. Luk, editor, Advanced signal processing algorithms, architectures, and implementations XII, volume 4791 of Proceedings of SPIE, pages 167-170, 2002. 
[41] M. Van Barel, R. Vandebril, and N. Mastronardi. The Lanczos-Ritz values appearing in an orthogonal similarity reduction of a matrix into semiseparable form. Report TW 360, Department of Computer Science, Katholieke Universiteit Leuven, Celestijnenlaan 200A, B-3001 Leuven (Heverlee), May 2003.

[42] E. Van Camp, N. Mastronardi, and M. Van Barel. Two fast algorithms for solving diagonal-plus-semiseparable linear systems. Tech. Rep. 17/2002, IAC/CNR, Bari, 2002 .

[43] R. Vandebril, M. Van Barel, and M. Mastronardi. An implicit QR algorithm for semiseparable matrices to compute the eigendecomposition of symmetric matrices. Report TW 367, Department of Computer Science, K.U.Leuven, Leuven, Belgium, August 2003.

[44] R. Vandebril, M. Van Barel, and M. Mastronardi. A QR method for computing the singular values via semiseparable matrices. Report TW 366, Department of Computer Science, K.U.Leuven, Leuven, Belgium, August 2003. 